\title{
Nonlinear Integrable Couplings of Levi Hierarchy and WKI Hierarchy
}

\author{
Zhengduo Shan, ${ }^{1,2,3,4}$ Hongwei Yang, ${ }^{5}$ and Baoshu Yin ${ }^{1,4}$ \\ ${ }^{1}$ Institute of Oceanology, Chinese Academy of Sciences, Qingdao 266071, China \\ ${ }^{2}$ School of Mathematics and Physics, Qingdao University of Science and Technology, Qingdao 266061, China \\ ${ }^{3}$ Graduate School, University of Chinese Academy of Sciences, Beijing 100049, China \\ ${ }^{4}$ Key Laboratory of Ocean Circulation and Wave, Chinese Academy of Sciences, Qingdao 266071, China \\ ${ }^{5}$ College of Mathematics and Systems Science, Shandong University of Science and Technology, Qingdao 266590, China
}

Correspondence should be addressed to Hongwei Yang; hwyang1979@163.com

Received 11 June 2014; Accepted 2 July 2014; Published 14 July 2014

Academic Editor: Yufeng Zhang

Copyright (c) 2014 Zhengduo Shan et al. This is an open access article distributed under the Creative Commons Attribution License, which permits unrestricted use, distribution, and reproduction in any medium, provided the original work is properly cited.

With the help of the known Lie algebra, a type of new 8-dimensional matrix Lie algebra is constructed in the paper. By using the 8-dimensional matrix Lie algebra, the nonlinear integrable couplings of the Levi hierarchy and the Wadati-Konno-Ichikawa (WKI) hierarchy are worked out, which are different from the linear integrable couplings. Based on the variational identity, the Hamiltonian structures of the above hierarchies are derived.

\section{Introduction}

The notion of integrable couplings was introduced when the study of Virasoro symmetric algebras $[1,2]$. To find as many new integrable systems and their integrable couplings as possible and to elucidate in depth their algebraic and geometric properties are of both theoretical and practical value. During the past few years, some interesting integrable couplings and associated properties of some known interesting integrable hierarchies, such as the Ablowitz-KaupNewell-Segur (AKNS) hierarchy and the Kaup-Newell (KN) hierarchy, were obtained [3-13]. Here it is necessary to point out that the above mentioned integrable couplings are linear for the supplementary variable, so they are called linear integrable couplings.

Recently, Professor Ma proposed the notion of nonlinear integrable couplings and gave the general scheme to construct nonlinear integrable couplings of hierarchies [14]. Based on the general scheme of constructing nonlinear integrable couplings, Professor Zhang introduced some new explicit Lie algebras and obtained the nonlinear integrable couplings of the Giachetti-Johnson (GJ) hierarchy, the Yang hierarchy, and the classical Boussinesq-Burgers (CBB) hierarchy $[15,16]$.
The aim of the paper is to seek the nonlinear integrable couplings of the Levi hierarchy and the WKI hierarchy as well as their Hamiltonian structures. The plan of the paper is as follows. In Section 2, with the help of the Lie algebra $G=\left\{\left(\begin{array}{cc}g_{1} & g_{2} \\ 0 & g_{1}+g_{2}\end{array}\right) \mid g_{1}, g_{2} \in s l(2)\right\}$, an 8-dimensional matrix Lie algebra is presented. It is different from the Lie algebras given in [14-16]. By employing the 8-dimensional matrix Lie algebra, the nonlinear integrable couplings of the Levi hierarchy and the WKI hierarchy are derived in Section 3. Furthermore, the corresponding Hamiltonian structures are worked out by virtue of the variational identity in Section 4 . Finally, some conclusions are obtained in Section 5.

\section{8-Dimensional Matrix Lie Algebra}

The Lie algebra is presented as $H=\operatorname{span}\left\{h_{1}, h_{2}, h_{3}, h_{4}\right\}$ with the basis as follows:

$$
\begin{array}{lll}
h_{1}=\left(\begin{array}{ll}
1 & 0 \\
0 & 0
\end{array}\right), & h_{2}=\left(\begin{array}{ll}
0 & 0 \\
0 & 1
\end{array}\right), \\
h_{3}=\left(\begin{array}{ll}
0 & 1 \\
0 & 0
\end{array}\right), & h_{4}=\left(\begin{array}{ll}
0 & 0 \\
1 & 0
\end{array}\right),
\end{array}
$$


equipped with the commutators

$$
\begin{aligned}
& {\left[h_{1}, h_{2}\right]=0, \quad\left[h_{1}, h_{3}\right]=h_{3}, \quad\left[h_{1}, h_{4}\right]=-h_{4},} \\
& {\left[h_{2}, h_{3}\right]=-h_{3}, \quad\left[h_{2}, h_{4}\right]=h_{4}, \quad\left[h_{3}, h_{4}\right]=h_{1}-h_{2} .}
\end{aligned}
$$

By virtue of the Lie algebra $H$, we construct an 8 -dimensional matrix Lie algebra

$$
G=\operatorname{span}\left\{g_{1}, g_{2}, g_{3}, g_{4}, g_{5}, g_{6}, g_{7}, g_{8}\right\}
$$

with the basis as follows:

$$
\begin{array}{ll}
g_{1}=\left(\begin{array}{cc}
h_{1} & 0 \\
0 & h_{1}
\end{array}\right), & g_{2}=\left(\begin{array}{cc}
h_{2} & 0 \\
0 & h_{2}
\end{array}\right), \\
g_{3}=\left(\begin{array}{cc}
h_{3} & 0 \\
0 & h_{3}
\end{array}\right), & g_{4}=\left(\begin{array}{cc}
h_{4} & 0 \\
0 & h_{4}
\end{array}\right), \\
g_{5}=\left(\begin{array}{ll}
0 & h_{1} \\
0 & h_{1}
\end{array}\right), & g_{6}=\left(\begin{array}{ll}
0 & h_{2} \\
0 & h_{2}
\end{array}\right), \\
g_{7}=\left(\begin{array}{ll}
0 & h_{3} \\
0 & h_{3}
\end{array}\right), & g_{8}=\left(\begin{array}{ll}
0 & h_{4} \\
0 & h_{4}
\end{array}\right),
\end{array}
$$

which have the commutative relations

$$
\begin{array}{ccc}
{\left[g_{1}, g_{2}\right]=0,} & {\left[g_{1}, g_{3}\right]=g_{3},} & {\left[g_{1}, g_{4}\right]=-g_{4},} \\
{\left[g_{2}, g_{3}\right]=-g_{3},} & {\left[g_{2}, g_{4}\right]=g_{4},} & {\left[g_{3}, g_{4}\right]=g_{1}-g_{2},} \\
{\left[g_{1}, g_{5}\right]=0,} & {\left[g_{1}, g_{6}\right]=0,} & {\left[g_{1}, g_{7}\right]=g_{7},} \\
{\left[g_{1}, g_{8}\right]=-g_{8},} & {\left[g_{2}, g_{5}\right]=0,} & {\left[g_{2}, g_{6}\right]=0,} \\
{\left[g_{2}, g_{7}\right]=-g_{7},} & {\left[g_{2}, g_{8}\right]=g_{8},} & {\left[g_{3}, g_{5}\right]=-g_{7},} \\
{\left[g_{3}, g_{6}\right]=g_{7},} & {\left[g_{3}, g_{7}\right]=0,} & {\left[g_{3}, g_{8}\right]=g_{5}-g_{6},} \\
{\left[g_{4}, g_{5}\right]=g_{8},} & {\left[g_{4}, g_{6}\right]=-g_{8},} & {\left[g_{4}, g_{7}\right]=g_{6}-g_{5},} \\
{\left[g_{4}, g_{8}\right]=0,} & {\left[g_{5}, g_{6}\right]=0,} & {\left[g_{5}, g_{7}\right]=g_{7},} \\
{\left[g_{5}, g_{8}\right]=-g_{8},} & {\left[g_{6}, g_{7}\right]=-g_{7},} & {\left[g_{6}, g_{8}\right]=g_{8},}
\end{array}
$$

Denoting $G_{1}=\operatorname{span}\left\{g_{1}, g_{2}, g_{3}, g_{4}\right\}$ and $G_{2}=\operatorname{span}\left\{g_{5}, g_{6}, g_{7}\right.$, $\left.g_{8}\right\}$, then we have

$$
G=G_{1} \oplus G_{2}, \quad G_{1} \cong H, \quad\left[G_{1}, G_{2}\right] \subset G_{2} .
$$

Here we need to emphasize that the subalgebras $G_{1}$ and $G_{2}$ are both nonsemisimple, which is very important for deriving nonlinear integrable couplings of hierarchies. By using the Lie algebra $G$, we can construct a few kinds of loop algebras $\widetilde{G}=$ $G \otimes \lambda^{N n+j}, N$ and $j$ stand for natural numbers. Among these loop algebras, the simplest one is

$$
\begin{array}{r}
\widetilde{G}=\operatorname{span}\left\{g_{i}(n)\right\}_{i=1}^{8}, \quad g_{i}(n)=g_{i} \lambda^{n}, \\
i=1,2,3,4,5,6,7,8,
\end{array}
$$

along with the commutators $\left[g_{i}(m), g_{j}(n)\right]=\left[g_{i}, g_{j}\right] \lambda^{m+n}$, $\operatorname{deg}\left(g_{i}(n)\right)=n, m, n \in Z, 1 \leq i$, and $j \leq 8$.

In this section, by virtue of the Lie algebra $H$, we construct an 8-dimensional matrix Lie algebra $G$ and corresponding loop algebra $\widetilde{G}$; in what follows we will generate the nonlinear integrable couplings of hierarchies by using the loop algebra $\widetilde{G}$.

\section{Nonlinear Integrable Couplings of Hierarchies}

In this section, based on the loop algebra $\widetilde{G}$, we construct two isospectral problems to generate the nonlinear integrable couplings of the Levi hierarchy and the WKI hierarchy, respectively.

3.1. Nonlinear Integrable Couplings of Levi Hierarchy. Take the following isospectral problem:

$$
\begin{gathered}
\phi_{x}=U \phi, \quad \lambda_{t}=0, \\
U=\frac{1}{2}\left(-\lambda+u_{1}-u_{2}\right)\left(g_{1}(0)-g_{2}(0)\right)+u_{1} g_{3}(0)+u_{2} g_{4}(0) \\
+\frac{1}{2}\left(u_{3}-u_{4}\right)\left(g_{5}(0)-g_{6}(0)\right)+u_{3} g_{7}(0)+u_{4} g_{8}(0) .
\end{gathered}
$$

Set $V=v_{1}\left(g_{1}(0)-g_{2}(0)\right)+v_{2} g_{3}(0)+v_{3} g_{4}(0)+v_{4}\left(g_{5}(0)-\right.$ $\left.g_{6}(0)\right)+v_{5} g_{7}(0)+v_{6} g_{8}(0)$, where $v_{i}=\sum_{m \geq 0} v_{i m} \lambda^{-m}, i=$ $1,2,3,4,5,6$. Solving the stationary zero curvature equation $V_{x}=[U, V]$ gives rise to the recursion relation as follows:

$$
\begin{gathered}
v_{1 m x}=u_{1} v_{3 m}-u_{2} v_{2 m}, \\
v_{2 m x}=-v_{2 m+1}+\left(u_{1}-u_{2}\right) v_{2 m}-2 u_{1} v_{1 m}, \\
v_{3 m x}=v_{3 m+1}-\left(u_{1}-u_{2}\right) v_{3 m}+2 u_{2} v_{1 m} \\
v_{4 m x}=u_{1} v_{6 m}-u_{4} v_{2 m}-u_{2} v_{5 m}+u_{3} v_{3 m}+u_{3} v_{6 m}-u_{4} v_{5 m}, \\
v_{5 m x}=\left(u_{1}-u_{2}\right) v_{5 m}-2 u_{3} v_{1 m}-v_{5 m+1}-2 u_{1} v_{4 m} \\
+\left(u_{3}-u_{4}\right)\left(v_{2 m}+v_{5 m}\right)-2 u_{3} v_{4 m}, \\
v_{6 m x}=2 u_{4} v_{1 m}-\left(u_{1}-u_{2}\right) v_{6 m}+v_{6 m+1}+2 u_{2} v_{4 m} \\
-\left(u_{3}-u_{4}\right)\left(v_{3 m}+v_{6 m}\right)+2 u_{4} v_{4 m}, \\
v_{10}=-\frac{\alpha}{2} \neq 0, \quad v_{20}=v_{30}=v_{40}=v_{50}=v_{60}=0 \\
v_{11}=0, \quad v_{21}=\alpha u_{1}, \quad v_{31}=\alpha u_{2},
\end{gathered}
$$$$
v_{41}=0, \quad v_{51}=\alpha u_{3}, \quad v_{61}=\alpha u_{4}, \quad v_{12}=\alpha u_{1} u_{2},
$$ 


$$
\begin{gathered}
v_{22}=\alpha u_{1}\left(u_{1}-u_{2}\right)-\alpha u_{1 x}, \\
v_{32}=\alpha u_{2}\left(u_{1}-u_{2}\right)+\alpha u_{2 x}, \\
v_{42}=\alpha\left(u_{1} u_{4}+u_{2} u_{3}+u_{3} u_{4}\right), \\
v_{52}=\alpha u_{3}\left(u_{1}-u_{2}\right)-\alpha u_{3 x}+\alpha\left(u_{1}+u_{3}\right)\left(u_{3}-u_{4}\right), \\
v_{62}=\alpha u_{4}\left(u_{1}-u_{2}\right)+\alpha u_{4 x}+\alpha\left(u_{2}+u_{4}\right)\left(u_{3}-u_{4}\right) .
\end{gathered}
$$

Denoting $V_{+}^{(n)}=\sum_{m=0}^{n}\left(v_{1 m}, v_{2 m}, v_{3 m}, v_{4 m}, v_{5 m}, v_{6 m}\right)^{T} \lambda^{n-m}$ and $V_{-}^{(n)}=\lambda^{n} V-V_{+}^{(n)}$, it is easy to compute

$$
\begin{aligned}
-V_{+x}^{(n)}+\left[U, V_{+}^{(n)}\right]= & v_{2 n+1} g_{3}(0)-v_{3 n+1} g_{4}(0) \\
& +v_{5 n+1} g_{7}(0)-v_{6 n+1} g_{8}(0) .
\end{aligned}
$$

Take

$$
\begin{gathered}
V^{(n)}=V_{+}^{(n)}+\Delta_{n} \\
\Delta_{n}=\frac{1}{2}\left(v_{2 n}-v_{3 n}-2 v_{1 n}\right)\left(g_{1}(0)-g_{2}(0)\right) \\
+\frac{1}{2}\left(v_{5 n}-v_{6 n}-2 v_{4 n}\right)\left(g_{5}(0)-g_{6}(0)\right) .
\end{gathered}
$$

Thus, the zero curvature equation

$$
U_{t}-V_{x}^{(n)}+\left[U, V^{(n)}\right]=0
$$

leads to the following integrable system:

$$
\begin{aligned}
& U_{t} \\
& =\left(\begin{array}{l}
u_{1} \\
u_{2} \\
u_{3} \\
u_{4}
\end{array}\right)_{t} \\
& =\left(\begin{array}{c}
-v_{2 n+1}+\left(v_{2 n}-v_{3 n}-2 v_{1 n}\right) u_{1} \\
v_{3 n+1}-\left(v_{2 n}-v_{3 n}-2 v_{1 n}\right) u_{2} \\
-v_{5 n+1}+\left(v_{2 n}-v_{3 n}-2 v_{1 n}\right) u_{3}+\left(v_{5 n}-v_{6 n}-2 v_{4 n}\right)\left(u_{1}+u_{3}\right) \\
v_{6 n+1}-\left(v_{2 n}-v_{3 n}-2 v_{1 n}\right) u_{4}-\left(v_{5 n}-v_{6 n}-2 v_{4 n}\right)\left(u_{2}+u_{4}\right)
\end{array}\right) \\
& =\left(\begin{array}{l}
v_{2 n x}-v_{1 n x} \\
v_{3 n x}+v_{1 n x} \\
v_{5 n x}-v_{4 n x} \\
v_{6 n x}+v_{4 n x}
\end{array}\right) \\
& =\left(\begin{array}{cccc}
0 & 0 & 0 & \partial \\
0 & 0 & \partial & 0 \\
0 & \partial & 0 & -\partial \\
\partial & 0 & -\partial & 0
\end{array}\right)\left(\begin{array}{c}
v_{1 n}+v_{3 n}+v_{4 n}+v_{6 n} \\
-v_{1 n}+v_{2 n}-v_{4 n}+v_{5 n} \\
v_{1 n}+v_{3 n} \\
-v_{1 n}+v_{2 n}
\end{array}\right)=J_{1} P_{n},
\end{aligned}
$$

where $J_{1}$ is a Hamiltonian operator and $P_{n+1}=L_{1} P_{n}$, the recurrence operator $L_{1}$ is given from (9) by

$$
L_{1}=\left(\begin{array}{cccc}
L_{11} & L_{12} & L_{13} & L_{14} \\
L_{21} & L_{22} & L_{23} & L_{24} \\
0 & 0 & L_{33} & L_{34} \\
0 & 0 & L_{43} & L_{44}
\end{array}\right)
$$

where

$$
\begin{aligned}
& L_{11}=\partial-\left(u_{2}+u_{4}\right)+\partial^{-1}\left(u_{1}+u_{3}\right) \partial, \\
& L_{12}=\left(u_{2}+u_{4}\right)+\partial^{-1}\left(u_{2}+u_{4}\right) \partial, \\
& L_{13}=-u_{4}+\partial^{-1} u_{3} \partial, \\
& L_{14}=u_{4}+\partial^{-1} u_{4} \partial, \\
& L_{21}=-\left(u_{1}+u_{3}\right)-\partial^{-1}\left(u_{1}+u_{3}\right) \partial, \\
& L_{22}=-\partial+\left(u_{1}+u_{3}\right)-\partial^{-1}\left(u_{2}+u_{4}\right) \partial, \\
& L_{23}=-u_{3}-\partial^{-1} u_{3} \partial, \\
& L_{24}=u_{3}-\partial^{-1} u_{4} \partial, \\
& L_{33}=\partial-u_{2}+\partial^{-1} u_{1} \partial, \\
& L_{34}=u_{2}+\partial^{-1} u_{2} \partial, \\
& L_{43}=-u_{1}-\partial^{-1} u_{1} \partial, \\
& L_{44}=u_{1}-\partial^{-1} u_{2} \partial-\partial .
\end{aligned}
$$

Therefore, the system (13) can be written as

$$
\left(\begin{array}{l}
u_{1} \\
u_{2} \\
u_{3} \\
u_{4}
\end{array}\right)_{t}=J_{1} L_{1}^{n-1}\left(\begin{array}{c}
v_{11}+v_{31}+v_{41}+v_{61} \\
-v_{11}+v_{21}-v_{41}+v_{51} \\
v_{11}+v_{31} \\
-v_{11}+v_{21}
\end{array}\right)
$$

When $u_{3}=u_{4}=0$, the system (13) reduces to the Levi hierarchy; therefore, in terms of the definition of integrable coupling, we conclude that the system (13) is an integrable coupling of the Levi hierarchy. Especially taking $n=2$, we have the following reduced equations:

$$
\begin{aligned}
& u_{1 t}=\alpha\left(u_{1}^{2}-2 u_{1} u_{2}\right)_{x}-\alpha u_{1 x x}, \\
& u_{2 t}=\alpha\left(-u_{2}^{2}+2 u_{1} u_{2}\right)_{x}+\alpha u_{2 x x}, \\
& u_{3 t}=2 \alpha\left(u_{1} u_{3}-u_{2} u_{3}-u_{1} u_{4}-u_{3} u_{4}\right)_{x}-\alpha u_{3 x x}+\alpha\left(u_{3}^{2}\right)_{x} \\
& u_{4 t}=2 \alpha\left(u_{1} u_{4}-u_{2} u_{4}+u_{3} u_{4}+u_{2} u_{3}\right)_{x}+\alpha u_{4 x x}-\alpha\left(u_{4}^{2}\right)_{x} .
\end{aligned}
$$

Obviously, (18) are nonlinear equations in $u_{3}$ and $u_{4}$, so we call (13) the nonlinear integrable coupling of the Levi hierarchy.

3.2. Nonlinear Integrable Couplings of WKI Hierarchy. Consider an isospectral problem

$$
\phi_{x}=U \phi, \quad \lambda_{t}=0,
$$




$$
\begin{aligned}
U= & i\left[g_{2}(1)-g_{1}(1)\right]+u_{1} g_{3}(1)+u_{2} g_{4}(1) \\
& +u_{3} g_{7}(1)+u_{4} g_{8}(1) .
\end{aligned}
$$

Set $V=\sum_{m \geq 0} \widetilde{V}$, where

$$
\begin{aligned}
\widetilde{V}=[ & \lambda v_{1 m} g_{1}(-m)-\lambda v_{1 m} g_{2}(-m)+\left(v_{2 m x}+i \lambda u_{1} v_{1 m}\right) \\
& \times g_{3}(-m)+\left(v_{3 m x}+i \lambda u_{2} v_{1 m}\right) g_{4}(-m) \\
& +\lambda v_{4 m} g_{5}(-m)-\lambda v_{4 m} g_{6}(-m) \\
& +\left(v_{5 m x}+i \lambda u_{3} v_{1 m}+i \lambda u_{1} v_{4 m}+i \lambda u_{3} v_{4 m}\right) g_{7}(-m) \\
& \left.+\left(v_{6 m x}+i \lambda u_{4} v_{1 m}+i \lambda u_{2} v_{4 m}+i \lambda u_{4} v_{4 m}\right) g_{8}(-m)\right]
\end{aligned}
$$

Because every term in $U$ includes $\lambda, V$ is different from the common form and includes potentials $u_{1}, u_{2}, u_{3}$, and $u_{4}$ and $v_{2 m x}, v_{3 m x}, v_{5 m x}, v_{6 m x}$, and so on. Then the zero curvature equation $V_{x}=[U, V]$ yields

$$
\begin{aligned}
& v_{1 m x}=u_{1} v_{3 m x}-u_{2} v_{2 m x}, \\
& i\left(u_{1} v_{1 m+1}\right)_{x}+v_{2 m x x}=-2 i v_{2 m+1 x}, \\
& i\left(u_{2} v_{1 m+1}\right)_{x}+v_{3 m x x}=2 i v_{3 m+1 x}, \\
& v_{4 m x}=u_{1} v_{6 m x}-u_{2} v_{5 m x}+u_{3} v_{3 m x}-u_{4} v_{2 m x} \\
& +u_{3} v_{6 m x}-u_{4} v_{5 m x} \\
& i\left(u_{3} v_{1 m+1}+u_{1} v_{4 m+1}+u_{3} v_{4 m+1}\right)_{x}+v_{5 m x x}=-2 i v_{5 m+1 x}, \\
& i\left(u_{4} v_{1 m+1}+u_{2} v_{4 m+1}+u_{4} v_{4 m+1}\right)_{x}+v_{6 m x x}=2 i v_{6 m+1 x} \\
& v_{10}=\alpha_{1}, \quad v_{20}=\alpha_{2}, \quad v_{30}=\alpha_{3}, \\
& v_{40}=\alpha_{4}, \quad v_{50}=\alpha_{5}, \quad v_{60}=\alpha_{6}, \\
& v_{11}=\frac{2}{p}, \quad v_{21}=\frac{-u_{1}}{p}, \quad v_{31}=\frac{u_{2}}{p}, \\
& v_{41}=-\frac{2}{p}-\frac{2}{p^{\prime}}, \quad v_{51}=\frac{u_{1}}{p}+\frac{u_{1}+u_{3}}{p^{\prime}}, \\
& v_{61}=-\frac{u_{2}}{p}-\frac{u_{2}+u_{4}}{p^{\prime}}, \quad p=\sqrt{1-u_{1} u_{2}} \\
& p^{\prime}=\sqrt{1-\left(u_{1}+u_{3}\right)\left(u_{2}+u_{4}\right)} .
\end{aligned}
$$

Denoting $V_{+}^{(n)}=\sum_{m=0}^{n} \widetilde{V}=\lambda^{n} V-V_{-}^{(n)}$, then we have $-V_{+x}^{(n)}+$ $\left[U, V_{+}^{(n)}\right]=V_{-x}^{(n)}-\left[U, V_{-}^{(n)}\right]$. A direct calculation reads

$$
\begin{aligned}
-V_{+x}^{(n)}+\left[U, V_{+}^{(n)}\right]= & -\lambda v_{2 n-1 x x} g_{3}(0)-\lambda v_{3 n-1 x x} g_{4}(0) \\
& -\lambda v_{5 n-1 x x} g_{7}(0)-\lambda v_{6 n-1 x x} g_{8}(0)
\end{aligned}
$$

Therefore, the zero curvature equation

$$
U_{t}-V_{x}^{(n)}+\left[U, V^{(n)}\right]=0
$$

admits

$$
\begin{aligned}
U_{t}= & \left(\begin{array}{l}
u_{1} \\
u_{2} \\
u_{3} \\
u_{4}
\end{array}\right)_{t}=\left(\begin{array}{c}
v_{2 n-1 x x} \\
v_{3 n-1 x x} \\
v_{5 n-1 x x} \\
v_{6 n-1 x x}
\end{array}\right)=\left(\begin{array}{cccc}
0 & 0 & 0 & \partial^{2} \\
0 & 0 & -\partial^{2} & 0 \\
0 & \partial^{2} & 0 & -\partial^{2} \\
-\partial^{2} & 0 & \partial^{2} & 0
\end{array}\right) \\
& \times\left(\begin{array}{c}
-v_{3 n-1}-v_{6 n-1} \\
v_{2 n-1}+v_{5 n-1} \\
-v_{3 n-1} \\
v_{2 n-1}
\end{array}\right)=J_{2} Q_{n-1} .
\end{aligned}
$$

Here $J_{2}$ is a Hamiltonian operator and $Q_{n}=L_{2} Q_{n-1}$, the recurrence operator $L_{2}$ is given from (21) by

$$
L_{2}=\left(\begin{array}{cccc}
L_{11} & L_{12} & L_{13} & L_{14} \\
L_{21} & L_{22} & L_{23} & L_{24} \\
0 & 0 & L_{33} & L_{34} \\
0 & 0 & L_{43} & L_{44}
\end{array}\right) \text {, }
$$

where

$$
\begin{aligned}
L_{11}= & -\frac{i}{2} \partial-\frac{i}{4} \frac{u_{2}+u_{4}}{p^{\prime}} \partial^{-1} \frac{u_{1}+u_{3}}{p^{\prime}} \partial^{2}, \\
L_{12}= & \frac{i}{4} \frac{u_{2}+u_{4}}{p^{\prime}} \partial^{-1} \frac{u_{2}+u_{4}}{p^{\prime}} \partial^{2}, \\
L_{13}= & -\frac{i}{4}\left[\frac{u_{2}+u_{4}}{p^{\prime}} \partial^{-1} \frac{1}{p^{\prime}}\left(-u_{1} \partial^{2}+q \partial \frac{q}{p} \partial^{-1} \frac{u_{1}}{p} \partial^{2}\right)\right. \\
& \left.+\frac{u_{2}+u_{4}}{p} \partial^{-1} \frac{u_{1}}{p} \partial^{2}\right], \\
L_{14}= & \frac{i}{4}\left[\frac{u_{2}+u_{4}}{p^{\prime}} \partial^{-1} \frac{1}{p^{\prime}}\left(-u_{2} \partial^{2}+q \partial \frac{q}{p} \partial^{-1} \frac{u_{2}}{p} \partial^{2}\right)\right. \\
& \left.+\frac{u_{2}+u_{4}}{p} \partial^{-1} \frac{u_{2}}{p} \partial^{2}\right],
\end{aligned}
$$




$$
\begin{aligned}
L_{21}= & -\frac{i}{4} \frac{u_{1}+u_{3}}{p^{\prime}} \partial^{-1} \frac{u_{1}+u_{3}}{p^{\prime}} \partial^{2}, \\
L_{22}= & \frac{i}{2} \partial+\frac{i}{4} \frac{u_{1}+u_{3}}{p^{\prime}} \partial^{-1} \frac{u_{2}+u_{4}}{p^{\prime}} \partial^{2}, \\
L_{23}= & -\frac{i}{4}\left[\frac{u_{1}+u_{3}}{p^{\prime}} \partial^{-1} \frac{1}{p^{\prime}}\left(-u_{1} \partial^{2}+q \partial \frac{q}{p} \partial^{-1} \frac{u_{1}}{p} \partial^{2}\right)\right. \\
L_{24}= & \frac{i}{4}\left[\frac{u_{1}+u_{3}}{p^{\prime}} \partial^{-1} \frac{1}{p^{\prime}} \partial^{2} \frac{1}{p^{\prime}} \partial^{-1}\right. \\
L_{44}= & \left.\frac{i}{2} \partial+\frac{u_{1}+u_{3}}{p} \partial^{-1} \frac{u_{1}}{p} \partial^{2}\right], \\
L_{43}= & -\frac{i}{4} \frac{u_{1}}{p} \partial^{-1} \frac{u_{1}}{p} \partial^{2}, \frac{1}{p} \frac{u_{2}}{p} \partial^{2} . \\
L_{34} & \left.\frac{i}{p} \frac{u_{2}}{p} \partial^{-1} \frac{u_{2}}{p} \partial^{2}+q \partial \frac{q}{p} \partial^{-1} \frac{u_{2}}{p} \partial^{2}\right) \\
L_{33}= & -\frac{i}{2} \partial-\frac{i}{4} \frac{u_{2}}{p} \partial^{-1} \frac{u_{1}}{p} \partial^{2}, \\
& \left.+\frac{u_{1}+u_{3}}{p} \partial^{-1} \frac{u_{2}}{p} \partial^{2}\right],
\end{aligned}
$$

Here $p, p^{\prime}$ are given in $(21)$ and $q=\sqrt{u_{1} u_{4}+u_{2} u_{3}+u_{3} u_{4}}$. Hence, the system (24) can be written as

$$
\left(\begin{array}{l}
u_{1} \\
u_{2} \\
u_{3} \\
u_{4}
\end{array}\right)_{t}=J_{2} L_{2}^{n-2}\left(\begin{array}{c}
v_{31}+v_{61} \\
v_{21}+v_{51} \\
v_{31} \\
v_{21}
\end{array}\right)
$$

When $u_{3}=u_{4}=0$, the system (24) is just the WKI hierarchy. Taking $n=2$, the system (24) reduces the following equations:

$$
\begin{gathered}
u_{1 t}=\left(-\frac{u_{1}}{\sqrt{1-u_{1} u_{2}}}\right)_{x x}, \\
u_{2 t}=\left(\frac{u_{2}}{\sqrt{1-u_{1} u_{2}}}\right)_{x x}, \\
u_{3 t}=\left(\frac{u_{1}}{\sqrt{1-u_{1} u_{2}}}+\frac{u_{1}+u_{3}}{\sqrt{1-\left(u_{1}+u_{3}\right)\left(u_{2}+u_{4}\right)}}\right)_{x x}, \\
u_{4 t}=\left(-\frac{u_{2}}{\sqrt{1-u_{1} u_{2}}}-\frac{u_{2}+u_{4}}{\sqrt{1-\left(u_{1}+u_{3}\right)\left(u_{2}+u_{4}\right)}}\right)_{x x} .
\end{gathered}
$$

It is easy to find that (28) are nonlinear equations in $u_{3}$ and $u_{4}$, so we call (24) the nonlinear integrable coupling of the WKI hierarchy.

\section{Hamiltonian Structures}

In this section, we will seek the Hamiltonian structures of the nonlinear integrable couplings of the Levi hierarchy (13) and the WKI hierarchy (24) by virtue of the variational identity. First, we construct a linear map $G \rightarrow R^{8}, g=\sum_{i=1}^{8} v_{i} g_{i}(0) \rightarrow$ $v=\left(v_{1}, v_{2}, v_{3}, v_{4}, v_{5}, v_{6}, v_{7}, v_{8}\right)^{T}, g \in G, v \in R^{8}$. We can conclude that the linear map is an isomorphism from $G$ to $R^{8}$. Let $a, b \in R^{8}$; matrix $R(b)$ is determined by [9]

$$
[a, b]^{T}=a^{T} R(b)
$$

where $a=\left(a_{1}, \ldots, a_{8}\right)^{T}$ and $b=\left(b_{1}, \ldots, b_{8}\right)^{T}$. From (29), we have

$$
R(b)=\left(\begin{array}{cccccccc}
0 & 0 & b_{3} & -b_{4} & 0 & 0 & b_{7} & -b_{8} \\
0 & 0 & -b_{3} & b_{4} & 0 & 0 & -b_{7} & b_{8} \\
b_{4} & -b_{4} & b_{2}-b_{1} & 0 & b_{8} & -b_{8} & b_{6}-b_{5} & 0 \\
-b_{3} & b_{3} & 0 & b_{1}-b_{2} & -b_{7} & b_{7} & 0 & b_{5}-b_{6} \\
0 & 0 & 0 & 0 & 0 & 0 & b_{3}+b_{7} & -b_{4}-b_{8} \\
0 & 0 & 0 & 0 & 0 & 0 & -b_{3}-b_{7} & b_{4}+b_{8} \\
0 & 0 & 0 & 0 & b_{4}+b_{8} & -b_{4}-b_{8} & b_{2}-b_{1}-b_{5}+b_{6} & 0 \\
0 & 0 & 0 & 0 & -b_{3}-b_{7} & b_{3}+b_{7} & 0 & b_{1}-b_{2}+b_{5}-b_{6}
\end{array}\right) .
$$


Solving the matrix equation for the constant matrix $F$, $R(b) F=-(R(b) F)^{T}, F^{T}=F$,

$$
F=\left(\begin{array}{llllllll}
1 & 0 & 0 & 0 & 1 & 0 & 0 & 0 \\
0 & 1 & 0 & 0 & 0 & 1 & 0 & 0 \\
0 & 0 & 0 & 1 & 0 & 0 & 0 & 1 \\
0 & 0 & 1 & 0 & 0 & 0 & 1 & 0 \\
1 & 0 & 0 & 0 & 0 & 0 & 0 & 0 \\
0 & 1 & 0 & 0 & 0 & 0 & 0 & 0 \\
0 & 0 & 0 & 1 & 0 & 0 & 0 & 0 \\
0 & 0 & 1 & 0 & 0 & 0 & 0 & 0
\end{array}\right) .
$$

Then in terms of $F$, define a linear functional in the $R^{8}$

$$
\begin{aligned}
\{a, b\}= & a^{T} F b=\left(a_{1}+a_{5}\right) b_{1}+\left(a_{2}+a_{6}\right) b_{2}+\left(a_{4}+a_{8}\right) b_{3} \\
& +\left(a_{3}+a_{7}\right) b_{4}+a_{1} b_{5}+a_{2} b_{6}+a_{4} b_{7}+a_{3} b_{8} .
\end{aligned}
$$

It is easy to find that $\{a, b\}$ satisfies the variational identity

$$
\frac{\delta}{\delta u} \int\left\{V, U_{\lambda}\right\} d x=\lambda^{-\gamma} \frac{\partial}{\partial \lambda} \lambda^{\gamma}\left\{V, U_{u}\right\} .
$$

Rewrite the Lax pair of nonlinear integrable coupling of the Levi hierarchy as follows:

$$
\begin{gathered}
U=\left(\frac{1}{2}\left(u_{1}-u_{2}-\lambda\right), \frac{1}{2}\left(\lambda-u_{1}+u_{2}\right), u_{1}, u_{2},\right. \\
\left.\frac{1}{2}\left(u_{3}-u_{4}\right), \frac{1}{2}\left(u_{4}-u_{3}\right), u_{3}, u_{4}\right)^{T}, \\
V=\left(v_{1},-v_{1}, v_{2}, v_{3}, v_{4},-v_{4}, v_{5}, v_{6}\right)^{T} .
\end{gathered}
$$

By using (32), we have

$$
\begin{gathered}
\left\{V, U_{\lambda}\right\}=-v_{1}-v_{4}, \quad\left\{V, U_{u_{1}}\right\}=v_{1}+v_{3}+v_{4}+v_{6}, \\
\left\{V, U_{u_{2}}\right\}=-v_{1}+v_{2}-v_{4}+v_{5}, \\
\left\{V, U_{u_{3}}\right\}=v_{1}+v_{3}, \quad\left\{V, U_{u_{4}}\right\}=-v_{1}+v_{2} .
\end{gathered}
$$

According the variational identity (33), we have

$$
\begin{aligned}
& \frac{\delta}{\delta u} \int\left(-v_{1}-v_{4}\right) d x \\
&=\lambda^{-\gamma} \frac{\partial}{\partial \lambda} \lambda^{\gamma}\left(v_{1}+v_{3}+v_{4}+v_{6},-v_{1}+v_{2}\right. \\
&\left.\quad-v_{4}+v_{5}, v_{1}+v_{3},-v_{1}+v_{2}\right)^{T} .
\end{aligned}
$$

Comparing the coefficients of $\lambda^{-n-2}$ yields

$$
\begin{aligned}
\frac{\delta}{\delta u} \int\left(-v_{1 n+1}-v_{4 n+1}\right) d x= & (\gamma-n) \\
& \times\left(\begin{array}{c}
v_{1 n}+v_{3 n}+v_{4 n}+v_{6 n} \\
-v_{1 n}+v_{2 n}-v_{4 n}+v_{5 n} \\
v_{1 n}+v_{3 n} \\
-v_{1 n}+v_{2 n}
\end{array}\right) .
\end{aligned}
$$

Taking $n=1$ gives rise to $\gamma=0$. Therefore,

$$
\begin{array}{r}
P_{n}=\frac{\delta H_{n}}{\delta u}, \quad H_{n}=\int \frac{a_{n+1}+d_{n+1}}{n} d x, \\
n \geq 1 .
\end{array}
$$

Hence, the nonlinear integrable coupling of the Levi hierarchy has the following Hamiltonian structure:

$$
U_{t}=J_{1} \frac{\delta H_{n}}{\delta u}, \quad n \geq 1
$$

Similar to (34), in order to deduce to the Hamiltonian structure of the nonlinear integrable coupling of the WKI hierarchy, we rewrite the Lax pair as follows:

$$
\begin{gathered}
U=\left(-i \lambda, i \lambda, \lambda u_{1}, \lambda u_{2}, 0,0, \lambda u_{3}, \lambda u_{4}\right)^{T} \\
V=\left(\lambda v_{1},-\lambda v_{1}, v_{2 x}+i \lambda u_{1} v_{1}, v_{3 x}+i \lambda u_{2} v_{1}, \lambda v_{4},\right. \\
-\lambda v_{4}, v_{5 x}+i \lambda u_{3}\left(v_{1}+v_{4}\right) \\
\left.+i \lambda u_{1} v_{4}, v_{6 x}+i \lambda u_{4}\left(v_{1}+v_{4}\right)+i \lambda u_{2} v_{4}\right)^{T} .
\end{gathered}
$$

Repeat the above procedure; we have

$$
\begin{gathered}
\left\{V, U_{\lambda}\right\}=-2 i\left(v_{1}+v_{4}\right)+2 i u_{1}\left(v_{3}+v_{6}\right) \\
-2 i u_{2}\left(v_{2}+v_{5}\right)+2 i u_{3} v_{3}-2 i u_{4} v_{2}, \\
\left\{V, U_{u_{1}}\right\}=2 i\left(v_{3}+v_{6}\right), \quad\left\{V, U_{u_{2}}\right\}=-2 i\left(v_{2}+v_{5}\right), \\
\left\{V, U_{u_{3}}\right\}=2 i v_{3}, \quad\left\{V, U_{u_{4}}\right\}=-2 i v_{2}, \\
\frac{\delta}{\delta u}\left\{2 i \left[-\left(v_{1 n-1}+v_{4 n-1}\right)+u_{1}\left(v_{3 n-1}+v_{6 n-1}\right)\right.\right. \\
\left.-u_{2}\left(v_{2 n-1}+v_{5 n-1}\right)+u_{3} v_{3 n-1}-u_{4} v_{2 n-1}\right] d x \\
=2 i\left(\begin{array}{c}
-v_{3 n-1}-v_{6 n-1} \\
v_{2 n-1}+v_{5 n-1} \\
-v_{3 n-1} \\
v_{2 n-1}
\end{array}\right) .
\end{gathered}
$$

Taking $n=2$ in above equation gives $\gamma=-1$. Therefore, $Q_{n-1}=\left(\delta \widetilde{H}_{n-1} / \delta u\right), n \geq 2$, where

$$
\begin{aligned}
\widetilde{H}_{n-1}=\int( & \left(v_{1 n-1}-v_{4 n-1}\right)-u_{1}\left(v_{3 n-1}-v_{6 n-1}\right) \\
& +u_{2}\left(v_{2 n-1}-v_{5 n-1}\right)-u_{3} v_{3 n-1} \\
& \left.+u_{4} v_{2 n-1}\right) \\
& \times(n-1)^{-1} d x
\end{aligned}
$$

Hence, the nonlinear integrable coupling of the WKI hierarchy has the following Hamiltonian structure:

$$
U_{t}=J_{2} \frac{\delta \widetilde{H}_{n-1}}{\delta u}, \quad n \geq 2 .
$$




\section{Conclusions}

In this paper, we presented a set of new 8-dimensional matrix Lie algebra by virtue of the Lie algebra given in [14-16]. With the help of the Lie algebra, we obtain the nonlinear integrable couplings of the Levi hierarchy and the WKI hierarchy. Their Hamiltonian structures are also worked out by the variational identity. The Lie algebra constructed in this paper can be used to generate the nonlinear integrable couplings of other hierarchies. We will study these problems in the future.

\section{Conflict of Interests}

The authors declare that there is no conflict of interests regarding the publication of this paper.

\section{Acknowledgment}

This work was supported by the Strategic Pioneering Program of Chinese Academy of Sciences (no. XDA 10020104), the Global Change and Air-Sea Interaction (no. GASI-03-01-0102), the Nature Science Foundation of Shandong Province of China (no. ZR2013AQ017), the Science and Technology Plan Project of Qingdao (no. 14-2-4-77-jch), the Open Fund of the Key Laboratory of Ocean Circulation and Waves, the Chinese Academy of Science (no. KLOCAW1401), the Open Fund of the Key Laboratory of Data Analysis and Application, and the State Oceanic Administration (no. LDAA-2013-04).

\section{References}

[1] W. X. Ma and B. Fuchssteiner, "Integrable theory of the perturbation equations," Chaos, Solitons and Fractals, vol. 7, no. 8, pp. 1227-1250, 1996.

[2] W. X. Ma, "Integrable couplings of soliton equations by perturbations-I. A general theory and application to the KdV hierarchy," Methods and Applications of Analysis, vol. 7, no. 1, pp. 21-55, 2000.

[3] Y. F. Zhang and F. K. Guo, "Two pairs of Lie algebras and the integrable couplings as well as the Hamiltonian structure of the Yang hierarchy," Chaos, Solitons and Fractals, vol. 34, no. 2, pp. 490-495, 2007.

[4] T. C. Xia, F. C. You, and W. Y. Zhao, "Multi-component Levi hierarchy and its multi-component integrable coupling system," Communications in Theoretical Physics, vol. 44, no. 6, pp. 990996, 2005.

[5] W. X. Ma, X. X. Xu, and Y. F. Zhang, "Semi-direct sums of Lie algebras and continuous integrable couplings," Physics Letters A, vol. 351, no. 3, pp. 125-130, 2006.

[6] Y. Zhang, "A generalized Boite-Pempinelli-Tu (BPT) hierarchy and its bi-Hamiltonian structure," Physics Letters A, vol. 317, no. 3-4, pp. 280-286, 2003.

[7] Y. F. Zhang and H. W. Tam, "New integrable couplings and Hamiltonian structure of the KN hierarchy and the DLW hierarchy," Communications in Nonlinear Science and Numerical Simulation, vol. 13, no. 3, pp. 524-533, 2008.

[8] H.-H. Dong and X.-Z. Wang, "A Lie algebra containing four parameters for the generalized Dirac hierarchy," Applied Mathematics and Computation, vol. 215, no. 2, pp. 459-463, 2009.
[9] F. Guo and Y. Zhang, "The quadratic-form identity for constructing the Hamiltonian structure of integrable systems," Journal of Physics A: Mathematical and General, vol. 38, no. 40, pp. 8537-8548, 2005.

[10] T. Xia, F. Yu, and Y. Zhang, "The multi-component coupled Burgers hierarchy of soliton equations and its multi-component integrable couplings system with two arbitrary functions," Physica A: Statistical Mechanics and Its Applications, vol. 343, no. 1-4, pp. 238-246, 2004.

[11] E. Fan and Y. Zhang, "Vector loop algebra and its applications to integrable system," Chaos, Solitons and Fractals, vol. 28, no. 4, pp. 966-971, 2006.

[12] W. X. Ma and Y. Zhang, "Component-trace identities for Hamiltonian structures," Applicable Analysis, vol. 89, no. 4, pp. 457-472, 2010.

[13] W. X. Ma and M. Chen, "Hamiltonian and quasi-Hamiltonian structures associated with semi-direct sums of Lie algebras," Journal of Physics A: Mathematical and General, vol. 39, no. 34, pp. 10787-10801, 2006.

[14] W. Ma, "Nonlinear continuous integrable Hamiltonian couplings," Applied Mathematics and Computation, vol. 217, no. 17, pp. 7238-7244, 2011.

[15] Y. Zhang, "Lie algebras for constructing nonlinear integrable couplings," Communications in Theoretical Physics, vol. 56, no. 5, pp. 805-812, 2011.

[16] Y. F. Zhang and B. L. Feng, "A few Lie algebras and their applications for generating integrable hierarchies of evolution types," Communications in Nonlinear Science and Numerical Simulation, vol. 16, no. 8, pp. 3045-3061, 2011. 


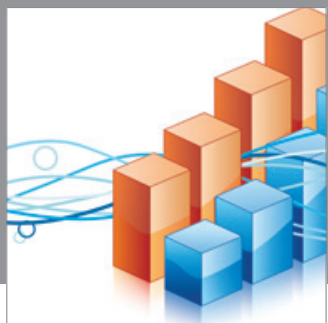

Advances in

Operations Research

mansans

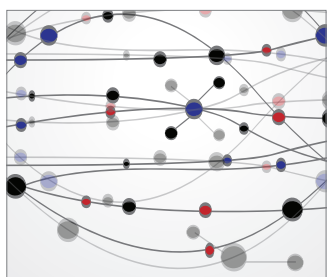

The Scientific World Journal
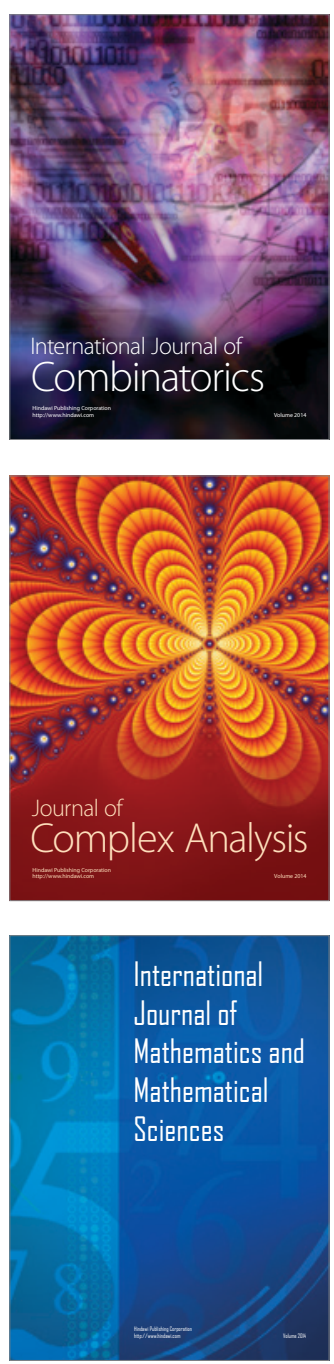
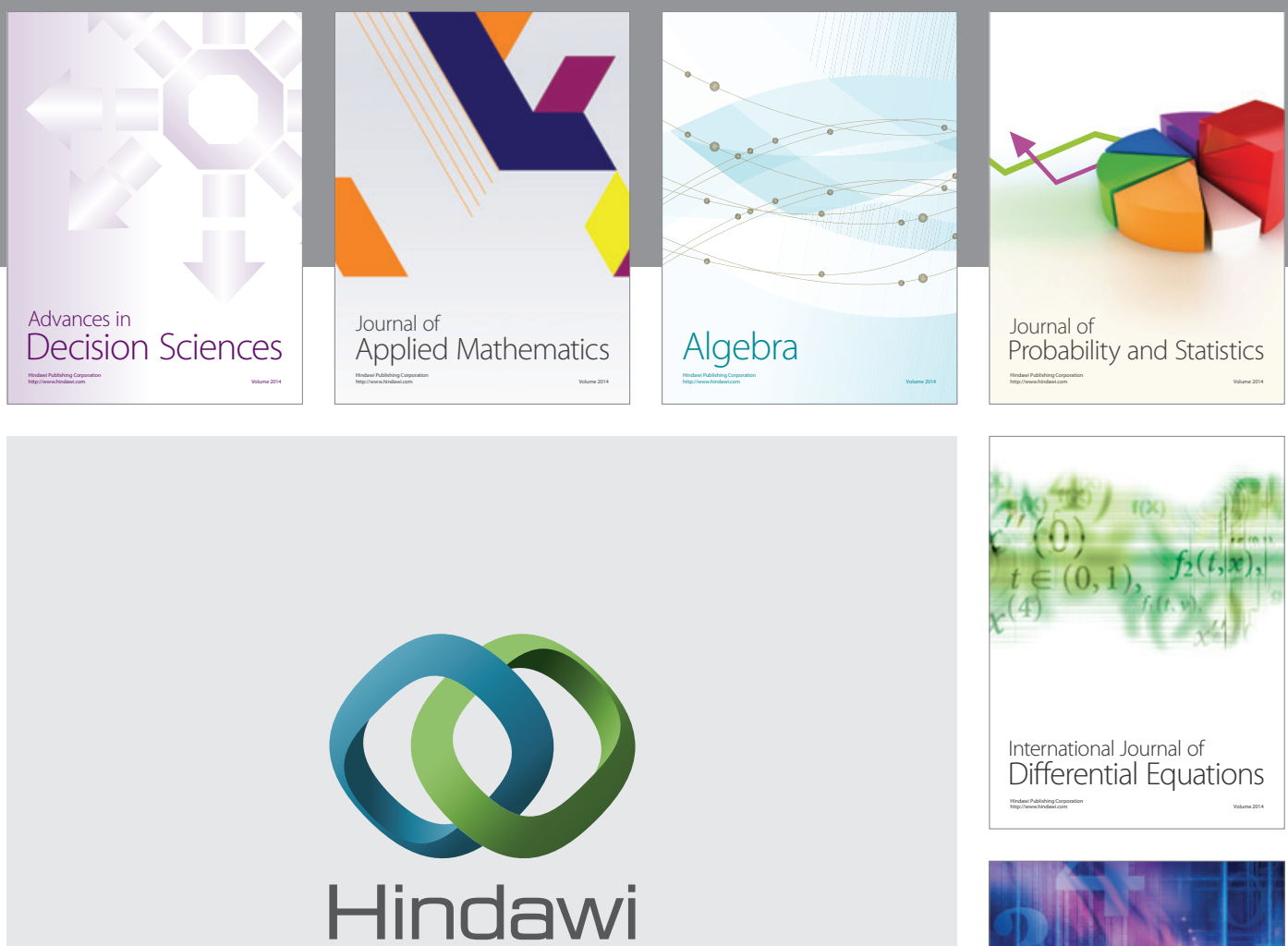

Submit your manuscripts at http://www.hindawi.com
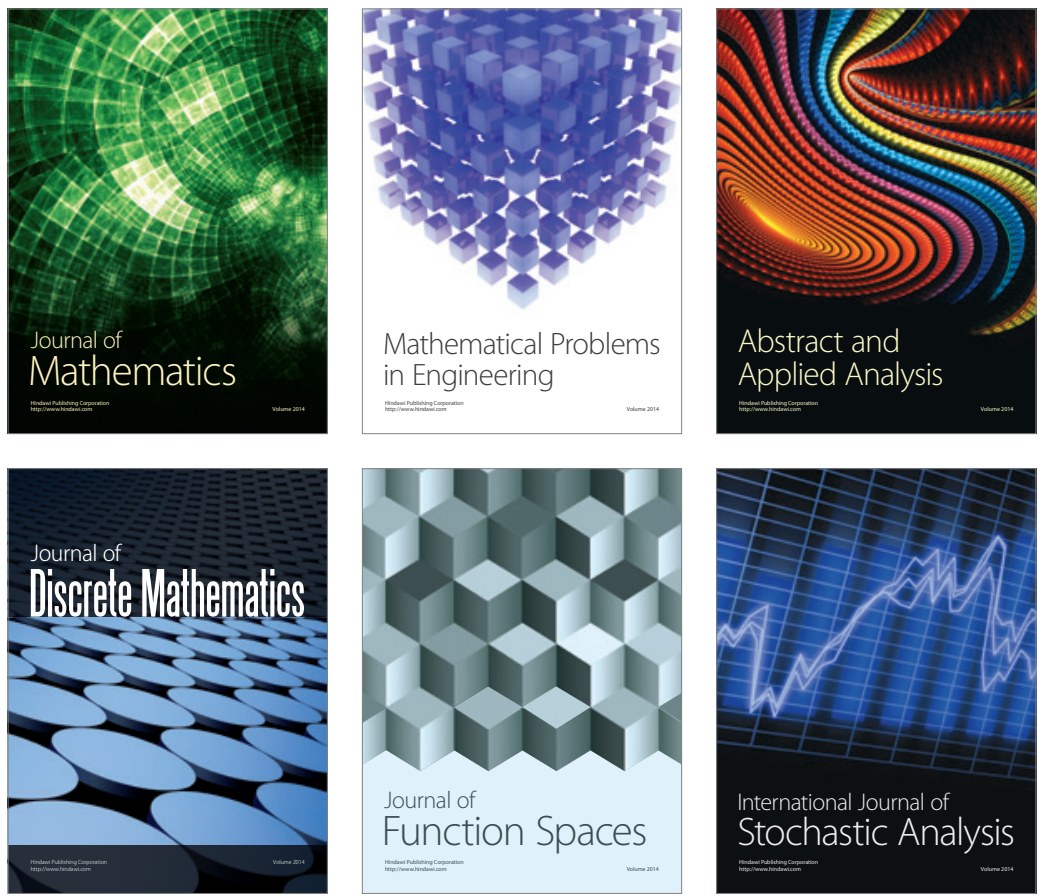

Journal of

Function Spaces

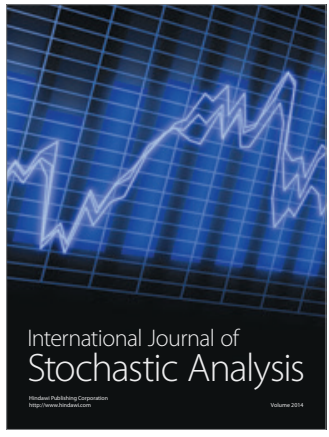

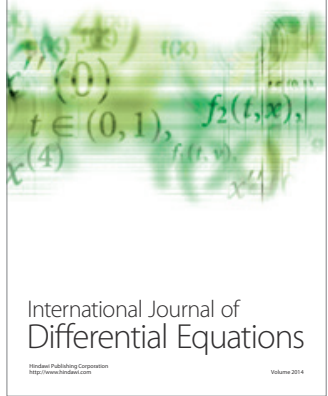
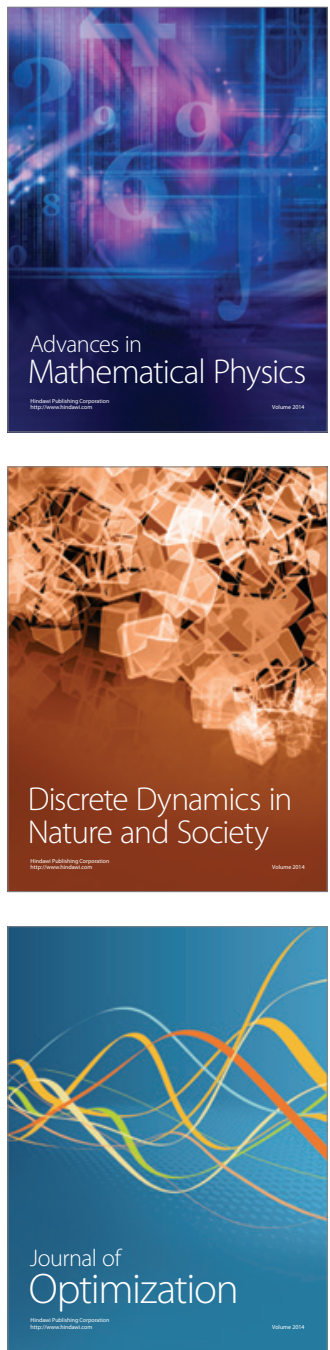